\title{
Neutrino masses in the Lee-Wick standard model
}

\author{
José Ramón Espinosa, ${ }^{1, *}$ Benjamín Grinstein, ${ }^{2, \dagger}$ Donal O’Connell, ${ }^{3, \ddagger}$ and Mark B. Wise ${ }^{3,8}$ \\ ${ }^{1}$ IFT-UAM/CSIC, Cantoblanco, 28049 Madrid, Spain \\ ${ }^{2}$ Department of Physics, University of California at San Diego, La Jolla, California 92093, USA \\ ${ }^{3}$ California Institute of Technology, Pasadena, California 91125, USA
}

(Received 17 December 2007; published 2 April 2008)

\begin{abstract}
Recently, an extension of the standard model based on ideas of Lee and Wick has been discussed. This theory is free of quadratic divergences and hence has a Higgs mass that is stable against radiative corrections. Here, we address the question of whether or not it is possible to couple very heavy particles, with masses much greater than the weak scale, to the Lee-Wick standard model degrees of freedom and still preserve the stability of the weak scale. We show that in the LW-standard model the familiar seesaw mechanism for generating neutrino masses preserves the solution to the hierarchy puzzle provided by the higher derivative terms. The very heavy right-handed neutrinos do not destabilize the Higgs mass. We give an example of new heavy degrees of freedom that would destabilize the hierarchy, and discuss a general mechanism for coupling other heavy degrees of freedom to the Higgs doublet while preserving the hierarchy.
\end{abstract}

DOI: 10.1103/PhysRevD.77.085002

PACS numbers: 12.90.+b, 12.15.Lk, 14.60.Pq

In a recent paper [1], ideas proposed by Lee and Wick $[2,3]$ were used to extend the standard model so that it does not contain quadratic divergences in the Higgs mass. Higher derivative kinetic terms for each of the standard model fields were added which improve the convergence of Feynman diagrams and give rise to a theory in which there are no quadratically divergent radiative corrections to the Higgs mass. The higher derivative terms induce new poles in the propagators of standard model fields which are interpreted as massive resonances. These resonances have wrong-sign kinetic terms which naively give rise to unacceptable instabilities. Lee and Wick propose altering the energy integrations in the definition of Feynman amplitudes so that the exponential growth does not occur. It appears that this can be done order by order in perturbation theory ${ }^{1}$ in a way which preserves unitarity. However, there is acausal behavior due to this deformation of the contour of integration. Physically this acausality is associated with the future boundary condition needed to forbid the exponentially growing modes. As long as the masses and widths of the LW-resonances are large enough, this acausality does not manifest itself on macroscopic scales and is not in conflict with experiment. The proposal to use Lee-Wick theory for the Higgs sector of the standard model was first presented in [7].

The massive resonances associated with the higher derivative terms in Lee-Wick theories have unusual properties. For example, they correspond to poles on the physical

\footnotetext{
*jose.espinosa@uam.es

†bgrinstein@ucsd.edu

donal@ theory.caltech.edu

${ }^{\S}$ wise@ theory.caltech.edu

${ }^{1}$ This is somewhat controversial. See [4-6].
}

sheet in scattering amplitudes. At the LHC, we may well discover new resonances, and it would be interesting to determine whether they are of normal or Lee-Wick type. This issue has recently been discussed in [8].

In the minimal standard model, the fermions get their masses through Yukawa couplings to the Higgs doublet. Gauge invariance forbids traditional mass terms. These Yukawa couplings do not give mass to the left-handed neutrinos. To describe neutrino masses, one can extend the particle content to include right-handed neutrinos. Right-handed neutrinos have no standard model gauge quantum numbers and so Majorana mass terms for them are allowed. If the right-handed neutrino Majorana masses are very large, we can understand the smallness of the observed neutrino masses, since the light neutrino masses scale as $m_{\nu} \sim v^{2} / m_{R}$, where $v$ is the vacuum expectation value for the Higgs doublet and $m_{R}$ is the mass scale associated with the right-handed neutrino Majorana masses. This attractive picture for the generation of neutrino masses is known as the seesaw mechanism [9].

Since the generation structure and the quarks are not the focus of this paper, let us simplify the notation by just considering a single standard model generation of leptons containing the left-handed doublet denoted by $L$ and the right-handed singlet $e_{R}$. Adding the right-handed neutrino $\nu_{R}$, the lepton sector of the standard model has Lagrange density,

$$
\begin{aligned}
\mathcal{L}= & \bar{L} i \not D L+\bar{e}_{R} i \not D e_{R}+\bar{\nu}_{R} i \not \partial \nu_{R}-\left(m_{R} \bar{\nu}_{R}^{c} \nu_{R}\right. \\
& \left.+g_{e} \bar{e}_{R} L H^{\dagger}+g_{Y} \bar{\nu}_{R} H^{T} \epsilon L+\text { H.c. }\right) .
\end{aligned}
$$

It was pointed out in [10] that the Feynman diagram in Fig. 1 gives a contribution to the mass term for the Higgs doublet that is quadratically divergent. If one uses dimen- 


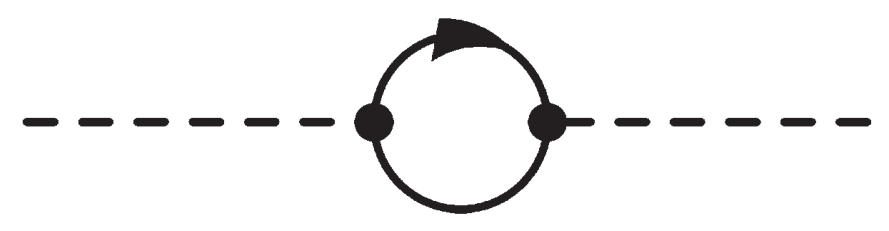

FIG. 1. One loop correction to the Higgs doublet mass. The dashed line represents the Higgs scalar, the solid arrowed line is the left-handed lepton, while the plain solid line is the righthanded neutrino.

sional regularization, which throws away quadratic divergences, there is still a finite correction

$$
\delta m_{H}^{2} \simeq-\frac{g_{Y}^{2}}{8 \pi^{2}} m_{R}^{2} \log \left(m_{R}^{2} / \mu^{2}\right)
$$

Since $g_{Y}$ and $m_{R}$ are constrained by $g_{Y}^{2} / m_{R} \approx m_{\nu} / v^{2}$, we see that the correction to the Higgs mass is too large when $m_{R} \gtrsim 10^{7} \mathrm{GeV}$ [10]. This is a manifestation of the hierarchy problem. In this paper we show that if one used the LW-standard model this does not occur. Even though the right-handed neutrinos are very heavy the higher derivative kinetic terms for the standard model fields are powerful enough to prevent the Higgs mass squared from getting a radiative correction that is proportional to $m_{R}^{2}$.

For simplicity, we gauge only $S U(2)_{W}$ so there is one set of gauge bosons, $\hat{A}_{\mu}^{A}$. The LW-standard model can be formulated either as a higher derivative theory, or as a theory without higher derivatives but with auxiliary LWfields. For the purposes of the present discussion, it is convenient to work with the higher derivative version of the theory. To emphasize that this is the LW-extended model, the fields with higher derivative kinetic terms are denoted by the presence of a hat. In this simplified version of the LW-standard model, the Lagrangian density is

$$
\begin{aligned}
& \mathcal{L}=-\frac{1}{2} \operatorname{tr} \hat{F}_{\mu \nu} \hat{F}^{\mu \nu}+\frac{1}{M_{A}^{2}} \operatorname{tr}\left(\hat{D}^{\mu} \hat{F}_{\mu \nu}\right)\left(\hat{D}^{\lambda} \hat{F}_{\lambda}{ }^{\nu}\right) \\
& +\left(\hat{D}_{\mu} \hat{H}\right)^{\dagger}\left(\hat{D}^{\mu} \hat{H}\right)-\frac{1}{M_{H}^{2}}\left(\hat{D}_{\mu} \hat{D}^{\mu} \hat{H}\right)^{\dagger}\left(\hat{D}_{\nu} \hat{D}^{\nu} \hat{H}\right) \\
& -V(\hat{H})+\overline{\hat{L}} i \hat{\not D} \hat{L}+\frac{1}{M_{L}^{2}} \overline{\hat{L}} i \not \hat{D} \not \hat{D} \hat{D} \hat{L}+\overline{\hat{e}}_{R} i \not \partial \hat{e}_{R} \\
& +\frac{1}{M_{E}^{2}} \overline{\hat{e}}_{R} i \not \partial \not \partial \hat{e}_{R}+\bar{\nu}_{R} i \not \partial \nu_{R}-\left(m_{R} \bar{\nu}_{R}^{c} \nu_{R}+g_{e} \overline{\hat{e}}_{R} \hat{L} \hat{H}^{\dagger}\right. \\
& \left.+g_{Y} \bar{\nu}_{R} \hat{H}^{T} \epsilon \hat{L}+\text { H.c. }\right) \text {. }
\end{aligned}
$$

Note that we have not added any higher derivative terms for the right-handed neutrino. Calculating the diagram in Fig. 1 in the LW-standard model and using a momentum cutoff $\Lambda$ to regularize the ultraviolet divergence, we find (neglecting the Lee-Wick mass parameter $M_{L}$ in comparison with $m_{R}$ and $\Lambda$ ) that

$$
\delta m_{\hat{H}}^{2}=-\frac{g_{Y}^{2}}{8 \pi^{2}} M_{L}^{2} \log \left(\frac{m_{R}^{2}+\Lambda^{2}}{m_{R}^{2}}\right) .
$$

This leads to acceptably small corrections to the Higgs mass if $g_{Y} M_{L} \lesssim 10 \mathrm{TeV}^{2}$. Thus, we have shown that, at one loop order, the Higgs mass is not destabilized by the presence of the right-handed neutrino. To go further, we will establish a power counting argument which shows that the divergence in the Higgs mass squared is at most logarithmic to all orders of perturbation theory. This is sufficient to show that there are no large finite corrections to the Higgs mass since we take $m_{R}$ of order the cutoff in our power counting.

To construct a perturbative power counting argument that shows to all orders in perturbation theory there is no quadratic divergence in the Higgs doublet mass term, we must fix a gauge in the higher derivative theory. We choose to add a covariant gauge fixing term $-\left(\partial_{\mu} \hat{A}^{A \mu}\right)^{2} / 2 \xi$ to the Lagrange density and introduce Faddeev-Popov ghosts that couple to the gauge bosons in the usual way. Then the propagator for the gauge field is

$$
\begin{aligned}
\hat{D}_{\mu \nu}^{A B}(p)= & \delta^{A B} \frac{-i}{p^{2}-p^{4} / M_{A}^{2}}\left(\eta_{\mu \nu}-(1-\xi) \frac{p_{\mu} p_{\nu}}{p^{2}}\right. \\
& \left.-\xi \frac{p_{\mu} p_{\nu}}{M_{A}^{2}}\right) .
\end{aligned}
$$

We work in Landau gauge, $\xi=0$, where the gauge boson propagator scales as $p^{-4}$ at high energy. The propagator for the Higgs scales at large momenta as $p^{-4}$ while the LWstandard model leptons, $\hat{L}$ and $\hat{e}_{R}$, have that scale as $p^{-3}$ at large momenta. Finally, the right-handed neutrino propagator and the Faddeev-Popov ghost propagator scales as $p^{-1}$ and $p^{-2}$, as usual. There are five kinds of vertices: those where only gauge bosons interact, vertices where gauge bosons interact with two scalars, and vertices where two ghosts interact with one gauge boson. A vertex where $n$ vectors interact (with no scalars) scales as $p^{6-n}$, a vertex with two scalars and $n$ vectors scales as $p^{4-n}$, while a vertex with two fermions and $n$ vectors scales as $p^{3-n}$. The vertex between two ghosts and one gauge field scales as one power of $p$, as usual, and the vertex from the Yukawa interaction of the Higgs doublet with the fermions has no factors of momentum.

Consider an arbitrary Feynman graph with $E$ external Higgs lines, $L$ loops, $I^{\prime}$ internal vector lines, $I$ internal scalar lines, $I_{R}$ internal right-handed neutrino lines, $I_{L}$ standard model lepton lines and $I_{g}$ internal ghost lines, and with $V_{n}^{\prime}$ vector self-interaction vertices, $V_{n}$ and $\bar{V}_{n}$ vertices with $n$ vectors and two scalar Higgs particles or left-handed leptons, respectively. We also suppose there are $V_{g}$ ghost vertices and $V_{Y}$ Yukawa vertices with two

\footnotetext{
${ }^{2}$ If we include a higher derivative term for the right-handed neutrino in Eq. (3), the correction to the Higgs mass is still proportional to $g_{Y} M_{L}$, leading to the same conclusion.
} 
fermions and a Higgs doublet. Then the superficial degree of divergence, $d$, is

$$
\begin{aligned}
d= & 4 L-4 I^{\prime}-4 I-I_{R}-3 I_{L}-2 I_{g}+\sum_{n} V_{n}^{\prime}(6-n) \\
& +\sum_{n} V_{n}(4-n)+\sum_{n} \bar{V}_{n}(3-n)+V_{g} .
\end{aligned}
$$

We can simplify this expression using some identities. First, the number of loops is related to the total number of propagators and vertices by

$$
\begin{aligned}
L= & I+I^{\prime}+I_{R}+I_{L}+I_{g}-\sum_{n}\left(V_{n}^{\prime}+V_{n}+\bar{V}_{n}\right) \\
& -V_{Y}-V_{g}+1,
\end{aligned}
$$

while the total number of lines entering or leaving the vertices is related to the number of propagators and external lines by

$$
\begin{aligned}
& \sum_{n}\left(n V_{n}^{\prime}+(n+2) V_{n}+(n+2) \bar{V}_{n}\right)+3 V_{g}+3 V_{Y} \\
& \quad=2\left(I+I^{\prime}+I_{R}+I_{L}+I_{g}\right)+E,
\end{aligned}
$$

where $E$ is the number of external scalars. Finally, we have the additional relations,

$$
\begin{aligned}
& 2 \sum_{n} V_{n}+V_{Y}=2 I+E, \quad 2 V_{g}=2 I_{g}, \\
& V_{Y}=2 I_{R}, \quad \sum_{n} \bar{V}_{n}+V_{Y}=I_{R}+I_{L} .
\end{aligned}
$$

With these identities in hand, we may express the superficial degree of divergence as

$$
d=6-2 L-V_{Y}-E .
$$

Scalar mass renormalizations have $E=2$. The only possible quadratic divergence in the scalar mass is at one loop with $V_{Y}=0$. As was discussed in [1], gauge invariance removes this potential quadratic divergence. Diagrams involving the leptons have at least $V_{Y}=2$ and so are at most logarithmically divergent. Diagrams with other external lines (which can be subdiagrams in the calculation of the Higgs mass term) can be analyzed similarly and do not change our conclusions.

We have shown that in at least one case it is possible to couple LW-standard model fields to degrees of freedom that are much heavier and still preserve the stability of the Higgs mass. Furthermore, this case is well motivated by the observed neutrino masses. However, this result is not true in general. Suppose, for example, there was a very heavy complex (normal) scalar $S$. An interaction term of the type $\mathcal{L}_{\text {int }}=g \hat{H}^{\dagger} \hat{H} S^{\dagger} S$ would lead to a large contribution to the Higgs boson mass. However, consider coupling the Higgs to a gauge singlet scalar $\hat{S}$ which has a higher derivative term in its Lagrange density:

$$
\mathcal{L}=\left(\partial_{\mu} \hat{S}\right)^{\dagger} \partial^{\mu} \hat{S}-M^{2} \hat{S}^{\dagger} \hat{S}-\frac{1}{m^{2}} \hat{S}^{\dagger} \partial^{4} \hat{S}+g \hat{H}^{\dagger} \hat{H} \hat{S}^{\dagger} \hat{S}
$$

Then the $\hat{S}$ propagator is given by

$$
\hat{D}=\frac{-i m^{2}}{p^{4}-p^{2} m^{2}+M^{2} m^{2}} .
$$

If we take the mass parameter $M$ to be large, as in the case of the scalar $S$, and choose the mass parameter $m$ to be of order of the weak scale, then the radiative corrections to the Higgs mass are still small despite the presence of the large scale $M$. The scalar $\hat{S}$ has unusual properties: for example, from the location of the poles in its propagator, one can see that it has a tree-level width which is large compared to its mass. We have not studied the consistency of this approach in detail.

In summary, we have shown in this paper that it is possible to couple the Lee-Wick standard model to physics at a much higher scale without destabilizing the Higgs mass. One of the best motivated examples of high scale physics is provided by experimental information on neutrino masses, and we find that the Lee-Wick standard model can easily be extended to incorporate a heavy right-handed neutrino without reintroducing fine-tuning of the Higgs mass. In addition, we have briefly described a scenario in which more general physics can be coupled to the Lee-Wick standard model while maintaining a naturally light Higgs.

We thank Andrew Cohen for stimulating discussions. J.R.E. is supported in part by CICYT, Spain, under Contract No. FPA2004-02015; by a Comunidad de Madrid project (P-ESP-00346); and by the European Commission under Contracts No. MRTN-CT-2004503369 and No. MRTN-CT-2006-035863. The work of B. G. was supported in part by the U.S. Department of Energy under Contract No. DE-FG03-97ER40546, while the work of D. O.C. and M. B. W. was supported in part by the U.S. Department of Energy under Contract No. DEFG03-92ER40701.
[1] B. Grinstein, D. O'Connell, and M. B. Wise, Phys. Rev. D 77, 025012 (2008).
[2] T. D. Lee and G. C. Wick, Nucl. Phys. B9, 209 (1969).

[3] T. D. Lee and G. C. Wick, Phys. Rev. D 2, 1033 (1970). 
[4] N. Nakanishi, Phys. Rev. D 3, 811 (1971).

[5] T. D. Lee and G. C. Wick, Phys. Rev. D 3, 1046 (1971).

[6] R.E. Cutkosky, P. V. Landshoff, D. I. Olive, and J.C. Polkinghorne, Nucl. Phys. B12, 281 (1969).

[7] K. Jansen, J. Kuti, and C. Liu, Phys. Lett. B 309, 119 (1993); 309, 127 (1993).

[8] T. G. Rizzo, J. High Energy Phys. 06 (2007) 070.

[9] M. Gell-Mann, P. Ramond, and R. Slansky, in Supergravity, edited by P. van Nieuwenhuizen and D.Z. Freedman (North-Holland, Amsterdam, 1979); T.
Yanagida, in Proceedings of the Workshop on the Baryon Number of the Universe and Unified Theories, Tsukuba, Japan, 1979; R. N. Mohapatra and G. Senjanovic, Phys. Rev. Lett. 44, 912 (1980); R. N. Mohapatra and G. Senjanovic, Phys. Rev. D 23, 165 (1981).

[10] J. A. Casas, J. R. Espinosa, and I. Hidalgo, J. High Energy Phys. 11 (2004) 057; F. Vissani, Phys. Rev. D 57, 7027 (1998); J. A. Casas, V. Di Clemente, A. Ibarra, and M. Quiros, Phys. Rev. D 62, 053005 (2000). 